
corn hybrids based on selection index. Horticultura Brasileira 39: 279-287. DOI: http://dx.doi.org/10.1590/s0102-0536-20210306.

\title{
Selecting experimental super sweet corn hybrids based on selection index
}

\author{
Welton Luiz Zaluski ${ }^{1 *} \mathbb{D}$; Marcos V Faria ${ }^{1 \mathbb{D}}$; Jocimar C Rosa ${ }^{2} \mathbb{D}$; Nathany R Chiquito ${ }^{1} \mathbb{D}$; Gabriela S de \\ Oliveira ${ }^{1} \mathbb{D}$; Vitor S Sagae ${ }^{1} \mathbb{D}$; Sidronio L da Silva Neto ${ }^{1 \mathbb{D}}$
}

${ }^{1}$ Universidade Estadual do Centro-Oeste, Departamento de Agronomia (UNICENTRO), Guarapuava-PR, Brasil; ${ }^{2}$ Universidade Estadual de Maringá, Departamento de Agronomia (UEM), Maringá-PR, Brasil (*Corresponding author)

\begin{abstract}
Super sweet corn hybrids shall present production and quality traits in order to meet farmer's, industry and consumer's expectations. The aim of this study was to select experimental super sweet corn hybrids based on the $\mathrm{Z}$ index $\left(I_{z}\right)$. We evaluated 64 experimental hybrids from crosses between inbred lines of different groups along with two check hybrids. The experiments were carried out in Guarapuava-PR in two sowing seasons in 2016. The evaluated traits were male flowering, ear length, ear diameter, soluble solids content, husked ear yield, unhusked ear yield, and grain yield. The traits which most contributed to the $\mathrm{Z}$ index were related to ear and grain yields and soluble solids content. The first sowing season was the most favorable for the expression of yield related traits. The experimental hybrid D3-30 x D5-46 showed high performance in both sowing seasons and the hybrid D2-17 x D5-46 stood out in the second sowing season, being both promising, showing high productivity and quality of ear, considering the $\mathrm{Z}$ index.
\end{abstract}

Keywords: Zea mays subsp. saccharata, ear yield, grain yield, Z index.

\section{RESUMO}

Seleção de híbridos experimentais de milho superdoce com base em índice de seleção

Híbridos de milho superdoce devem reunir características de produção e qualidade que atendam às expectativas dos agricultores, da indústria e do consumidor. O objetivo deste trabalho foi selecionar híbridos experimentais de milho superdoce com auxílio do índice de seleção Z (IZ). Foram avaliados 64 híbridos experimentais provenientes de cruzamentos entre 16 linhagens de grupos distintos, juntamente com dois híbridos testemunhas. Os experimentos foram conduzidos em Guarapuava-PR em duas épocas de semeadura no ano de 2016. Foram avaliados o florescimento masculino, comprimento e diâmetro de espiga, teor de sólidos solúveis, rendimento de espigas empalhadas, rendimento de espigas despalhadas e rendimento de grãos. As características que mais contribuíram para o índice Z (IZ) foram as relacionadas ao rendimento de espigas, rendimento de grãos e teor de sólidos solúveis. A primeira época foi a mais favorável para a expressão dos caracteres de rendimento. O híbrido experimental D3-30 x D5-46 teve bom desempenho em ambas as épocas e o híbrido D2-17 x D5-46 se destacou na segunda época de semeadura, sendo promissores, selecionados com alta produtividade e qualidade de espiga, considerando o índice Z.

Palavras-chave: Zea mays subsp. saccharata, rendimento de espigas, rendimento de grãos, índice $Z$.

Received on August 17, 2020; accepted on June 14, 2021

$\mathrm{S}$ weet and super sweet corn (Zea mays subsp. saccharatta) result from spontaneous mutations which alter the metabolic pathways of starch synthesis, providing higher sugar content in the kernels (Solomon et al., 2011). The super sweet corn is marketed and consumed mainly in canned form after industrial processing, adding greater value to this vegetable (Dovale et al., 2011; Favarato et al., 2016).

Sweet and super sweet corn breeding programs do not have well-defined heterotic patterns, unlike what occurs in common corn breeding programs. Heterosis effects are significant for the main traits related to productivity (Solomom et al., 2011; Kuki et al., 2017; Yuwono et al., 2017); however, almost always they are not sufficient for obtaining commercially successful sweet corn hybrids. This is due to the fact that traits such as ear diameter, ear length and soluble solids contents in grains, as well as high productivity, are essential to launch hybrids on the market (Souza Neto, 2019).

Due to a great number of traits which shall be evaluated, selection indexes based on linear combinations of variables can be efficient to assist in the simultaneous selection of traits (Candido et al., 2020), aiming to maximize the genetic gains, increasing the probability of success of the breeding program. Several studies used selection indexes in corn (Amaral Júnior et al., 2010; Freitas et al., 2013; Vieira et al., 2017; Lima et al., 2018) and some recent studies reported the use of different selection indexes in sweet corn genotypes (Silva et al, 2020a, b).

The $\mathrm{Z}$ index is obtained using the sum of standard values of evaluated variables. This index allows to select the most promising hybrids considering the set of traits of greatest interest. Thus, it is possible to generate more information 
on the phenotypical performance of the evaluated genotypes (Mendes et al., 2009; Wu et al., 2015), being unnecessary the use of estimates of genetic and phenotypic covariances (Ramalho et al., 2012).

The aim of this study was to select experimental super sweet corn hybrids using traits related to productivity and quality of ears based on the selection $\mathrm{Z}$ index $\left(I_{z}\right)$.

\section{MATERIAL AND METHODS}

The experiments were carried out in Guarapuava-PR $\left(25^{\circ} 21^{\prime} \mathrm{S}, 51^{\circ} 30^{\prime} \mathrm{W}\right.$, $1100 \mathrm{~m}$ altitude). The soil is classified as Typical Bruno Dystroferric Orison, very clayey texture (Nitsche et al., 2019).

Sixty four experimental super sweet corn hybrids were obtained from crosses between inbred lines of two different groups. Group I was formed by eight inbred lines from populations D2 (inbred lines D2-17 and D2-74), D3 (inbred lines D3-20, D3-30, D3-33 and D3-39) and D4 (inbred lines D4-20 and D4-59) and group II was formed by eight inbred lines from D5 population (inbred lines D5-09, D5-41, D5-42, D5-43, D5-46, D5-48, D5-53 and D555). The experimental hybrids were evaluated along with two marketable super sweet corn hybrids sh2sh2 used as checks, BRS Vivi (Embrapa) and Tropical Plus (Syngenta).

The genotypes were evaluated in two seasons, whose sowings were performed on October 15 and December 17, 2016. A randomized complete block design with three replications was used. Each plot consisted of one 5-m row, spacing $0.80 \mathrm{~m}$ between rows, with final stand corresponding to 62,500 plants $\mathrm{ha}^{-1}$. Grains were harvested when reached the milky stage, presenting $73 \%$ to $78 \%$ of moisture.

The following traits were evaluated in each plot: 1) male flowering (MF, in days), calculated from the sowing date up to pollen release by the anthers of the middle third of the tassel in $50 \%$ of the plants; 2) ear length (EL, in $\mathrm{cm}$ ): using a graduated ruler measuring samples of five husked ears; 3 ) ear diameter $(E D$, in $\mathrm{mm}$ ), with the aid of a digital caliper, measuring samples of five husked ears; 4) soluble solids content (SS, ${ }^{\circ}$ Brix): using three samples of grain mass, obtained with the aid of a digital refractometer, during harvesting; 5) husked ear yield (HEY, in $t \mathrm{ha}^{-1}$ ), calculated using the mass of all husked ears; 6) unhusked ear yield (UEY, in t $\mathrm{ha}^{-1}$ ), calculated using the mass of all the unhusked ears; 7) grain yield (GY, in $\left.t \mathrm{ha}^{-1}\right)$, related to the mass of grains cut close to the cob.

Data were submitted to Lilliefors \& Barlett tests and, after verifying the normality and homoscedasticity, individual and joint analyses of variance for these evaluated traits were performed. The means were grouped by Scott Knott test, using GENES software (Cruz, 2013).

The $\mathrm{Z}$ index $\left(I_{Z}\right)$ was used to help select the hybrids and identify the contribution of each trait on the genotype's selection. The $\mathrm{Z}$ index $\left(I_{Z}\right)$ was obtained using the sum of the $Z_{i j}$ standardized values of the seven evaluated traits (Mendes et al., 2009). Data standardization for each trait was obtained using the estimator $Z_{i j}=\left(Y_{i j}-\right.$ m) $/ S_{j}$, in which: $Z_{i j}$ is the standardized value of the trait in the hybrid $i(i=1$, $2, \ldots 64)$ in the replication $j(j=1,2,3)$; $Y_{i j}$ is the observed value of the trait in hybrid $i$ in replication $j ; m$ is the general average of the trait in replication $j$; and $S_{j}$ is the standard deviation of the trait in replication $j$.

For male flowering, in which the aim is to reduce the means, the values of the standardized variable $Z_{i j}$ were multiplied by -1 , in order to reverse the order, so that the positive values of the standardized variables are desirable for all traits. We also summed the value 3.0 to all estimates of the $Z_{i j}$ standardized variables in order to avoid the occurrence of negative values, considering this value as the populationaverage of each standardized trait.

Afterwards, we summed standardized $Z_{i j}$ values of the seven traits for each hybrid, in each replication, obtaining the $\mathrm{Z}$ index $\left(I_{Z}\right)$, which was considered a new variable. $I_{Z}$ values were submitted to individual and joint analysis of variance. The highest $I_{Z}$ values, associated with the smallest variances, were favorable for the selection of best hybrids.

\section{RESULTS AND DISCUSSION}

We noticed significant $(\mathrm{p}<0.05)$ hybrids $\mathrm{x}$ seasons interaction for male flowering (MF), ear length (EL), ear diameter (ED), soluble solids content (SS), husked ear yield (HEY), unkusked ear yield (UEY) and grain yield (GY).

Environmental influence on the expression of all the evaluated traits was highlighted by the significant effect of sowing seasons $(\mathrm{p}<0.01)$. Santos et al. (2014) evaluated super sweet corn genotype's performance and also obtained significant environmental effects. The hybrids $x$ seasons interaction changes the ranking of genotypes according to each environment, interfering with the recommendation of hybrids (Hallauer \& Miranda Filho, 2010; Abe \& Adelegan, 2019).

The coefficients of variation ranged from $1.70 \%$ (MF in the second season) to $15.65 \%$ ( $\mathrm{GY}$ in the first season) (Tables 1 and 2), whose values are considered suitable in experiments that evaluated corn cultivars (Gurgel et al., 2013).

We verified variability between genotypes considering the evaluated traits, being possible to select superior hybrids, based on the average of both sowing seasons or individually (Vencovsky, 1978).

Means of MF showed that the cycle of most experimental hybrids was late compared to checks (Table 1). Hybrid Tropical Plus showed the lowest value for MF (83 days) in the first season, which did not differ $(p>0.01)$ from the other twelve experimental hybrids whose MF occurred between 85 and 87 days after sowing date (Table 2). In the second season, occurred prolongation of the cycle of the experimental hybrids and checks (Table 1).

For male flowering (MF) the selection should focus on shortercycle hybrids, which make it possible to maximize profitability, anticipating the availability of the area for other crops. The results obtained showed that the average MF favored to reduce the cycle when comparing with other 
Table 1. Means of male flowering (MF), ear length (EL), ear diameter (ED) and soluble solids content (SS) of super sweet corn experimental hybrids and checks evaluated in two sowing seasons in 2016/2017 in Guarapuava-PR. Guarapuava, UNICENTRO, 2021.

\begin{tabular}{|c|c|c|c|c|c|c|c|c|}
\hline \multirow{3}{*}{$\begin{array}{l}\text { Genotype } \\
\text { D2-17 x D5-09 }\end{array}$} & \multicolumn{2}{|c|}{ MF (days) } & \multicolumn{2}{|c|}{ EL (cm) } & \multicolumn{2}{|c|}{ ED (mm) } & \multicolumn{2}{|c|}{ SS ( $\left({ }^{\circ}\right.$ Brix $)$} \\
\hline & Season 1 & Season 2 & Season 1 & Season 2 & Season 1 & Season 2 & Season 1 & Season 2 \\
\hline & $86 \mathrm{~d} \mathrm{~B}$ & $101 \mathrm{~b} \mathrm{~A}$ & $18.4 \mathrm{a} \mathrm{A}$ & $18.2 \mathrm{a} \mathrm{A}$ & $50.8 \mathrm{~b} \quad \mathrm{~A}$ & $50.4 \mathrm{~b} \mathrm{~A}$ & $13.9 \mathrm{c} \mathrm{B}$ & $15.5 \mathrm{~d} \mathrm{~A}$ \\
\hline D2-17 x D5-41 & 89 c B & $101 \mathrm{~b} \mathrm{~A}$ & $17.5 \mathrm{~b} \quad \mathrm{~A}$ & $16.6 \mathrm{a} \mathrm{A}$ & $49.3 \mathrm{~b} \quad \mathrm{~A}$ & $47.5 \mathrm{c} \mathrm{A}$ & $15.0 \mathrm{~b} \mathrm{~A}$ & $13.0 \mathrm{e} \mathrm{B}$ \\
\hline D2-17 x D5-42 & $90 \mathrm{~b} \mathrm{~B}$ & 104 a A & $17.4 \mathrm{~b} \mathrm{~A}$ & $16.3 \mathrm{a} \mathrm{A}$ & $48.0 \mathrm{c} \mathrm{A}$ & $46.9 \mathrm{c} \mathrm{A}$ & $16.5 \mathrm{a} \mathrm{A}$ & $16.5 \mathrm{c} \mathrm{A}$ \\
\hline D2-17 x D5-43 & $87 \mathrm{~d} \mathrm{~B}$ & $102 \mathrm{~b} \mathrm{~A}$ & $19.3 \mathrm{a} \mathrm{A}$ & 16.9 a $B$ & $49.8 \mathrm{~b} \mathrm{~A}$ & $46.4 \mathrm{c} \mathrm{B}$ & $14.2 \mathrm{c} \mathrm{A}$ & $15.8 \mathrm{~d} \mathrm{~A}$ \\
\hline D2-17 x D5-46 & $86 \mathrm{~d} \mathrm{~B}$ & $102 \mathrm{~b} \mathrm{~A}$ & $18.1 \mathrm{a} \mathrm{A}$ & $16.0 \mathrm{a} \mathrm{B}$ & $48.2 \mathrm{c} \mathrm{A}$ & $48.4 \mathrm{c} \mathrm{A}$ & 15.9 a B & $18.3 \mathrm{a} \mathrm{A}$ \\
\hline D2-17 x D5-48 & 93 a $B$ & 105 a A & $17.6 \mathrm{~b} \mathrm{~A}$ & $15.6 \mathrm{~b} \quad \mathrm{~B}$ & $45.0 \mathrm{c} \mathrm{A}$ & $47.6 \mathrm{c} \mathrm{A}$ & $14.3 \mathrm{c} \mathrm{B}$ & $18.3 \mathrm{~b} \mathrm{~A}$ \\
\hline D2-17 x D5-53 & $87 \mathrm{~d} \mathrm{~B}$ & $102 \mathrm{~b} \mathrm{~A}$ & $17.3 \mathrm{~b} \mathrm{~A}$ & $15.3 \mathrm{~b} \quad \mathrm{~B}$ & $48.7 \mathrm{c} \mathrm{A}$ & $47.0 \mathrm{c} \mathrm{A}$ & $14.0 \mathrm{c} \mathrm{B}$ & $16.7 \mathrm{c} \mathrm{A}$ \\
\hline D2-17 x D5-55 & $87 \mathrm{~d} \mathrm{~B}$ & 105 a A & $18.5 \mathrm{a} \mathrm{A}$ & $15.7 \mathrm{~b} \quad \mathrm{~B}$ & $49.4 \mathrm{~b} \mathrm{~A}$ & $48.4 \mathrm{c} \mathrm{A}$ & $14.5 \mathrm{~b} \mathrm{~B}$ & $19.1 \mathrm{a} \mathrm{A}$ \\
\hline D2-74 x D5-09 & $90 \mathrm{~b} \quad \mathrm{~B}$ & $103 \mathrm{~b} \mathrm{~A}$ & $19.3 \mathrm{a} \mathrm{A}$ & $17.5 \mathrm{a} \quad \mathrm{B}$ & $46.8 \mathrm{c} \quad \mathrm{B}$ & $51.3 \mathrm{~b} \mathrm{~A}$ & $12.8 \mathrm{c} \mathrm{B}$ & $17.4 \mathrm{~b} \mathrm{~A}$ \\
\hline D2-74 x D5-41 & 89 c B & $102 \mathrm{~b} \mathrm{~A}$ & $18.5 \mathrm{a} \mathrm{A}$ & $17.0 \mathrm{a} \mathrm{B}$ & $46.8 \mathrm{c} \mathrm{A}$ & $49.5 \mathrm{~b} \mathrm{~A}$ & 13.9 c B & $15.7 \mathrm{~d} \mathrm{~A}$ \\
\hline D2-74 x D5-42 & $91 \mathrm{~b} \quad \mathrm{~B}$ & 105 a $\mathrm{A}$ & $18.2 \mathrm{a} \mathrm{A}$ & 15.9 a $\mathrm{B}$ & $48.0 \mathrm{c} \mathrm{A}$ & $48.1 \mathrm{c} \mathrm{A}$ & $14.6 \mathrm{~b} \mathrm{~A}$ & $15.1 \mathrm{~d} \mathrm{~A}$ \\
\hline D2-74 x D5-43 & $87 \mathrm{~d} B$ & 104 a A & $18.8 \mathrm{a} \mathrm{A}$ & 16.2 a B & 48.9 c A & $45.7 \mathrm{c} \mathrm{A}$ & $13.2 \mathrm{c} \mathrm{B}$ & $18.4 \mathrm{~b} \mathrm{~A}$ \\
\hline D2-74 x D5-46 & 88 c B & $103 \mathrm{~b} \mathrm{~A}$ & $19.5 \mathrm{a} \mathrm{A}$ & $16.4 \mathrm{a} \quad \mathrm{B}$ & $50.9 \mathrm{~b} \quad \mathrm{~A}$ & $48.4 \mathrm{c} \mathrm{A}$ & $16.2 \mathrm{a} \mathrm{A}$ & $15.2 \mathrm{~d} \mathrm{~A}$ \\
\hline D2-74 x D5-48 & 94 a B & 105 a A & $19.1 \mathrm{a} \mathrm{A}$ & $16.4 \mathrm{a} \quad \mathrm{B}$ & $48.5 \mathrm{c} \mathrm{A}$ & $49.0 \mathrm{c} \mathrm{A}$ & $16.1 \mathrm{a} \mathrm{A}$ & $17.6 \mathrm{~b} \mathrm{~A}$ \\
\hline D2-74 x D5-53 & $91 \mathrm{~b} \quad \mathrm{~B}$ & 104 a A & $16.7 \mathrm{~b} \quad \mathrm{~A}$ & $15.0 \mathrm{~b} \quad \mathrm{~B}$ & $44.4 \mathrm{c} \mathrm{A}$ & $47.2 \mathrm{c} \mathrm{A}$ & $13.5 \mathrm{c} \mathrm{B}$ & $18.1 \mathrm{~b} \mathrm{~A}$ \\
\hline D2-74 x D5-55 & $90 \mathrm{~b} \quad \mathrm{~B}$ & 105 a A & $17.6 \mathrm{~b} \mathrm{~A}$ & $16.0 \mathrm{a} \mathrm{B}$ & $46.6 \mathrm{c} \mathrm{A}$ & $48.4 \mathrm{c} \mathrm{A}$ & $15.5 \mathrm{a} \mathrm{A}$ & $16.8 \mathrm{c} \mathrm{A}$ \\
\hline D3-20 x D5-09 & 88 c B & $103 \mathrm{~b} A$ & $19.2 \mathrm{a} \mathrm{A}$ & $16.2 \mathrm{a} \quad \mathrm{B}$ & $50.7 \mathrm{~b} \quad \mathrm{~A}$ & $51.3 \mathrm{a} \mathrm{A}$ & $14.8 \mathrm{~b} \mathrm{~A}$ & $16.1 \mathrm{~d} \mathrm{~A}$ \\
\hline D3-20 x D5-41 & 88 c B & $101 \mathrm{~b} \mathrm{~A}$ & $19.3 \mathrm{a} \mathrm{A}$ & $16.0 \mathrm{a} \mathrm{B}$ & $49.0 \mathrm{c} \mathrm{A}$ & $49.4 \mathrm{~b} \mathrm{~A}$ & $15.0 \mathrm{~b} \mathrm{~A}$ & $15.6 \mathrm{~d} \mathrm{~A}$ \\
\hline D3-20 x D5-42 & $91 \mathrm{~b} \quad \mathrm{~B}$ & 104 a A & $19.0 \mathrm{a} \mathrm{A}$ & $15.4 \mathrm{~b} \quad \mathrm{~B}$ & $48.3 \mathrm{c} \mathrm{A}$ & $48.4 \mathrm{c} \mathrm{A}$ & $16.5 \mathrm{a} \mathrm{A}$ & $17.1 \mathrm{c} \mathrm{A}$ \\
\hline D3-20 x D5-43 & $86 \mathrm{~d} B$ & $101 \mathrm{~b} \quad \mathrm{~A}$ & 18.9 a $\mathrm{A}$ & $15.8 \mathrm{~b} \quad \mathrm{~B}$ & $45.4 \mathrm{c} \mathrm{B}$ & $50.3 \mathrm{~b} \mathrm{~A}$ & 12.9 c B & $14.6 \mathrm{e} \mathrm{A}$ \\
\hline D3-20 x D5-46 & 88 c B & $102 \mathrm{~b} A$ & 19.8 a A & 16.7 a $B$ & $50.9 \mathrm{~b} \quad \mathrm{~A}$ & $50.7 \mathrm{~b} A$ & $16.1 \mathrm{a} \mathrm{A}$ & $17.0 \mathrm{c} \mathrm{A}$ \\
\hline D3-20 x D5-48 & 94 a B & 106 a A & $17.7 \mathrm{~b} \quad \mathrm{~A}$ & $16.4 \mathrm{a} \mathrm{A}$ & $46.6 \mathrm{c} \mathrm{B}$ & $50.7 \mathrm{~b}$ A & $13.5 \mathrm{c} \mathrm{B}$ & $17.9 \mathrm{~b} \mathrm{~A}$ \\
\hline D3-20 x D5-53 & 89 c B & 105 a A & $18.5 \mathrm{a} \mathrm{A}$ & $15.8 \mathrm{~b} \quad \mathrm{~B}$ & $47.5 \mathrm{c} \quad \mathrm{A}$ & $47.2 \mathrm{c} \mathrm{A}$ & $13.6 \mathrm{c} \mathrm{A}$ & $12.1 \mathrm{f} \mathrm{A}$ \\
\hline D3-20 x D5-55 & $86 \mathrm{~d} \mathrm{~B}$ & $103 \mathrm{~b} \mathrm{~A}$ & 18.7 a A & $16.6 \mathrm{a} \mathrm{B}$ & 48.5 c B & 52.9 a $\mathrm{A}$ & $15.1 \mathrm{~b} \quad \mathrm{~B}$ & $16.7 \mathrm{c} \mathrm{A}$ \\
\hline D3-30 x D5-09 & 88 c B & $102 \mathrm{~b} \mathrm{~A}$ & $19.2 \mathrm{a} \mathrm{A}$ & $16.0 \mathrm{a} \mathrm{B}$ & $53.3 \mathrm{a} \mathrm{A}$ & 48.8 c B & $15.5 \mathrm{a} \mathrm{A}$ & $15.1 \mathrm{~d} \mathrm{~A}$ \\
\hline D3-30 x D5-41 & 89 c B & $101 \mathrm{~b} \mathrm{~A}$ & $17.4 \mathrm{~b} \mathrm{~A}$ & $16.8 \mathrm{a} \mathrm{A}$ & $51.1 \mathrm{~b} \quad \mathrm{~A}$ & $50.1 \mathrm{~b} \mathrm{~A}$ & 15.9 a A & $15.9 \mathrm{~d} \mathrm{~A}$ \\
\hline D3-30 x D5-42 & $90 \mathrm{~b} \quad \mathrm{~B}$ & 104 a A & $17.7 \mathrm{~b} \quad \mathrm{~A}$ & $17.0 \mathrm{a} \mathrm{A}$ & $50.5 \mathrm{~b} \quad \mathrm{~A}$ & $47.3 \mathrm{c} \mathrm{A}$ & $17.1 \mathrm{a} \mathrm{A}$ & $18.0 \mathrm{~b} \mathrm{~A}$ \\
\hline D3-30 x D5-43 & 88 c B & $101 \mathrm{~b} A$ & $19.5 \mathrm{a} \mathrm{A}$ & $16.0 \mathrm{a} \mathrm{B}$ & $49.8 \mathrm{~b} \quad \mathrm{~A}$ & $49.0 \mathrm{~b} \mathrm{~A}$ & 16.9 a $\mathrm{A}$ & $12.6 \mathrm{e} \mathrm{B}$ \\
\hline D3-30 x D5-46 & $86 \mathrm{~d} B$ & 104 a A & 19.9 a A & 16.9 a B & 54.9 a A & $49.1 \mathrm{~b} \mathrm{~B}$ & $17.2 \mathrm{a} \mathrm{A}$ & $17.1 \mathrm{c} \mathrm{A}$ \\
\hline D3-30 x D5-48 & 93 a $B$ & 106 a A & $18.1 \mathrm{a} \mathrm{A}$ & $15.4 \mathrm{~b} \quad \mathrm{~B}$ & $51.6 \mathrm{~b} \quad \mathrm{~A}$ & $48.7 \mathrm{c} \mathrm{A}$ & 15.4 a B & $18.0 \mathrm{~b} \quad \mathrm{~A}$ \\
\hline D3-30 x D5-53 & 88 c B & 104 a A & $17.7 \mathrm{~b} \quad \mathrm{~A}$ & 16.2 a $B$ & $49.6 \mathrm{~b} \quad \mathrm{~A}$ & $48.5 \mathrm{c} \mathrm{A}$ & $14.5 \mathrm{~b} \mathrm{~B}$ & $16.8 \mathrm{c} \mathrm{A}$ \\
\hline D3-30 x D5-55 & $86 \mathrm{~d} \mathrm{~B}$ & 104 a A & $18.5 \mathrm{a} \mathrm{A}$ & $15.4 \mathrm{~b} \quad \mathrm{~B}$ & $47.9 \mathrm{c} \mathrm{A}$ & $47.1 \mathrm{c} \mathrm{A}$ & $14.2 \mathrm{c} \mathrm{B}$ & $17.6 \mathrm{~b} \mathrm{~A}$ \\
\hline D3-33 x D5-09 & $91 \mathrm{~b} B$ & $102 \mathrm{~b} \mathrm{~A}$ & $17.7 \mathrm{~b} \quad \mathrm{~A}$ & $15.3 \mathrm{~b} \quad \mathrm{~B}$ & $48.1 \mathrm{c} \mathrm{A}$ & $48.1 \mathrm{c} \mathrm{A}$ & $14.5 \mathrm{~b} \mathrm{~B}$ & $17.0 \mathrm{c} \mathrm{A}$ \\
\hline D3-33 x D5-41 & 89 c B & $102 \mathrm{~b} \mathrm{~A}$ & $17.3 \mathrm{~b} \quad \mathrm{~A}$ & $15.8 \mathrm{~b} \quad \mathrm{~B}$ & $50.7 \mathrm{~b} \quad \mathrm{~A}$ & $50.3 \mathrm{~b} \mathrm{~A}$ & 16.9 a A & 14.0 e $B$ \\
\hline D3-33 x D5-42 & $91 \mathrm{~b} B$ & 105 a A & $17.1 \mathrm{~b} \quad \mathrm{~A}$ & $15.8 \mathrm{~b} \quad \mathrm{~A}$ & 46.9 c A & $46.7 \mathrm{c} \mathrm{A}$ & $16.0 \mathrm{a} \mathrm{A}$ & $17.6 \mathrm{~b} \mathrm{~A}$ \\
\hline D3-33 x D5-43 & $87 \mathrm{~d} B$ & $103 \mathrm{~b} \quad \mathrm{~A}$ & $17.6 \mathrm{~b} \mathrm{~A}$ & $16.7 \mathrm{a} \quad \mathrm{A}$ & $46.7 \mathrm{c} \mathrm{A}$ & $48.4 \mathrm{c} \mathrm{A}$ & $14.1 \mathrm{c} \mathrm{A}$ & $10.6 \mathrm{f} \mathrm{B}$ \\
\hline D3-33 x D5-46 & 88 c B & $103 \mathrm{~b} \mathrm{~A}$ & 18.8 a A & $14.7 \mathrm{~b} \quad \mathrm{~B}$ & $49.8 \mathrm{~b} \quad \mathrm{~A}$ & $47.0 \mathrm{c} \mathrm{A}$ & $15.0 \mathrm{~b} \mathrm{~B}$ & $17.8 \mathrm{~b} \quad \mathrm{~A}$ \\
\hline D3-33 x D5-48 & 94 a B & 108 a A & $16.8 \mathrm{~b} \mathrm{~A}$ & 15.9 a A & $48.4 \mathrm{c} \mathrm{A}$ & $49.1 \mathrm{~b} \mathrm{~A}$ & 15.4 a B & $18.3 \mathrm{~b} \quad \mathrm{~A}$ \\
\hline D3-33 x D5-53 & $90 \mathrm{~b} \quad \mathrm{~B}$ & 105 a A & $17.8 \mathrm{~b} \quad \mathrm{~A}$ & 15.9 a $\mathrm{B}$ & $48.5 \mathrm{c} \mathrm{A}$ & $49.3 \mathrm{~b} \mathrm{~A}$ & $15.2 \mathrm{~b} \mathrm{~A}$ & $16.6 \mathrm{c} \mathrm{A}$ \\
\hline D3-33 x D5-55 & $91 \mathrm{~b} \quad \mathrm{~B}$ & 104 a A & $16.7 \mathrm{~b} \quad \mathrm{~A}$ & $15.3 \mathrm{~b} \quad \mathrm{~B}$ & $45.8 \mathrm{c} \mathrm{A}$ & $48.9 \mathrm{c} \mathrm{A}$ & 15.4 a B & $17.6 \mathrm{~b} \mathrm{~A}$ \\
\hline D3-39 x D5-09 & $92 \mathrm{~b} \quad \mathrm{~B}$ & $103 \mathrm{~b} A$ & $17.3 \mathrm{~b} \quad \mathrm{~A}$ & $16.0 \mathrm{a} \mathrm{A}$ & $48.8 \mathrm{c} \mathrm{A}$ & $47.4 \mathrm{c} \mathrm{A}$ & 15.6 a B & 18.6 a A \\
\hline D3-39 x D5-41 & $90 \mathrm{~b} B$ & $103 \mathrm{~b} A$ & $18.3 \mathrm{a} \mathrm{A}$ & $15.3 \mathrm{~b} \quad \mathrm{~B}$ & $48.2 \mathrm{c} \mathrm{A}$ & $46.0 \mathrm{c} \mathrm{A}$ & 13.8 c B & $18.1 \mathrm{~b} \mathrm{~A}$ \\
\hline D3-39 x D5-42 & $92 \mathrm{~b} \quad \mathrm{~B}$ & 105 a A & $18.2 \mathrm{a} \mathrm{A}$ & $16.1 \mathrm{a} \quad \mathrm{B}$ & $49.8 \mathrm{~b} \quad \mathrm{~A}$ & $49.4 \mathrm{~b} \mathrm{~A}$ & $16.2 \mathrm{a} \mathrm{A}$ & $17.5 \mathrm{~b} \quad \mathrm{~A}$ \\
\hline D3-39 x D5-43 & 89 c B & $103 \mathrm{~b} A$ & $19.0 \mathrm{a} \mathrm{A}$ & $16.3 \mathrm{a} \quad \mathrm{B}$ & 47.7 c A & $50.1 \mathrm{~b} \mathrm{~A}$ & $16.0 \mathrm{a} \mathrm{A}$ & 18.8 a B \\
\hline D3-39 x D5-46 & $86 \mathrm{~d} \mathrm{~B}$ & $101 \mathrm{~b} A$ & $18.7 \mathrm{a} A$ & $16.0 \mathrm{a} \quad \mathrm{B}$ & $49.9 \mathrm{~b} \quad \mathrm{~A}$ & $48.2 \mathrm{c} \mathrm{A}$ & $15.1 \mathrm{~b} \quad \mathrm{~A}$ & $16.6 \mathrm{c} \mathrm{A}$ \\
\hline D3-39 x D5-48 & 95 a $B$ & 108 a A & $18.6 \mathrm{a} \mathrm{A}$ & $16.3 \mathrm{a} \quad \mathrm{B}$ & $48.6 \mathrm{c} \mathrm{A}$ & $47.6 \mathrm{c} \mathrm{A}$ & 15.9 a $\mathrm{A}$ & $17.3 \mathrm{~b} \quad \mathrm{~A}$ \\
\hline D3-39 x D5-53 & 89 c B & 104 a A & $17.6 \mathrm{~b} \mathrm{~A}$ & $16.2 \mathrm{a} \quad \mathrm{B}$ & $48.4 \mathrm{c} \mathrm{A}$ & $48.4 \mathrm{c} \mathrm{A}$ & 15.7 a $\mathrm{A}$ & $17.2 \mathrm{~b} \quad \mathrm{~A}$ \\
\hline D3-39 x D5-55 & 89 c B & 104 a A & $18.1 \mathrm{a} \mathrm{A}$ & $15.0 \mathrm{~b} \quad \mathrm{~B}$ & $48.4 \mathrm{c} \mathrm{A}$ & $49.5 \mathrm{~b} \mathrm{~A}$ & $16.8 \mathrm{a} \mathrm{A}$ & $17.7 \mathrm{~b} \quad \mathrm{~A}$ \\
\hline D4-20 x D5-09 & $92 \mathrm{~b} \quad \mathrm{~B}$ & $102 \mathrm{~b} \mathrm{~A}$ & $18.4 \mathrm{a} \mathrm{A}$ & $16.2 \mathrm{a} \quad \mathrm{B}$ & $49.6 \mathrm{~b} \quad \mathrm{~A}$ & $49.9 \mathrm{~b} \mathrm{~A}$ & 13.9 c B & 18.7 a $\mathrm{A}$ \\
\hline D4-20 x D5-41 & $90 \mathrm{~b} \quad \mathrm{~B}$ & 105 a A & $17.6 \mathrm{~b} \mathrm{~A}$ & $16.0 \mathrm{a} \quad \mathrm{B}$ & $52.1 \mathrm{a} \quad \mathrm{A}$ & $46.3 \mathrm{c} \mathrm{B}$ & $17.0 \mathrm{a} \mathrm{A}$ & $16.6 \mathrm{c} \mathrm{A}$ \\
\hline
\end{tabular}


Table 1 continuation

\begin{tabular}{|c|c|c|c|c|c|c|c|c|c|c|}
\hline \multirow{3}{*}{$\begin{array}{l}\text { Genotype } \\
\text { D4-20 x D5-42 }\end{array}$} & \multicolumn{2}{|c|}{ MF (days) } & \multicolumn{4}{|c|}{ EL $(\mathbf{c m})$} & \multicolumn{2}{|c|}{ ED $(\mathbf{m m})$} & \multicolumn{2}{|c|}{ SS ( ${ }^{\circ}$ Brix) } \\
\hline & Season 1 & Season 2 & \multicolumn{2}{|c|}{ Season 1} & \multicolumn{2}{|c|}{ Season 2} & Season 1 & \multirow{2}{*}{$\begin{array}{c}\text { Season } 2 \\
49.3 \mathrm{~b} \mathrm{~A}\end{array}$} & Season 1 & Season 2 \\
\hline & $92 \mathrm{~b} \quad \mathrm{~B}$ & $105 \mathrm{a} \mathrm{A}$ & $17.3 \mathrm{~b}$ & $\mathrm{~A}$ & $16.1 \mathrm{a}$ & $\mathrm{A}$ & $49.0 \mathrm{c} \mathrm{A}$ & & 16.7 a A & $17.2 \mathrm{~b} \mathrm{~A}$ \\
\hline D4-20 x D5-43 & 89 c B & 105 a A & $18.4 \mathrm{a}$ & $\mathrm{A}$ & $16.1 \mathrm{a}$ & B & $47.9 \mathrm{c} \mathrm{A}$ & $47.5 \mathrm{c} \mathrm{A}$ & $16.8 \mathrm{a} \mathrm{A}$ & 13.9 e B \\
\hline D4-20 x D5-46 & $85 \mathrm{~d} \mathrm{~B}$ & $103 \mathrm{~b} \mathrm{~A}$ & $19.2 \mathrm{a}$ & A & $16.2 \mathrm{a}$ & B & $49.7 \mathrm{~b} \quad \mathrm{~A}$ & $48.5 \mathrm{c} \mathrm{A}$ & 15.9 a A & $16.7 \mathrm{c} \mathrm{A}$ \\
\hline D4-20 x D5-48 & 94 a B & 106 a A & $16.9 \mathrm{~b}$ & A & $15.0 \mathrm{~b}$ & B & $45.2 \mathrm{c} \mathrm{A}$ & $46.1 \mathrm{c} \mathrm{A}$ & $14.2 \mathrm{c} \mathrm{B}$ & $16.2 \mathrm{c} \mathrm{A}$ \\
\hline D4-20 x D5-53 & $90 \mathrm{~b}$ B & 104 a A & $16.4 \mathrm{~b}$ & $\mathrm{~A}$ & $15.4 \mathrm{~b}$ & A & $50.1 \mathrm{~b} \quad \mathrm{~A}$ & $49.9 \mathrm{~b} \mathrm{~A}$ & $14.8 \mathrm{~b} \mathrm{~B}$ & $17.4 \mathrm{~b} \quad \mathrm{~A}$ \\
\hline D4-20 x D5-55 & $90 \mathrm{~b} B$ & 105 a A & $18.0 \mathrm{~b}$ & A & $15.6 \mathrm{~b}$ & B & $48.2 \mathrm{c} \mathrm{A}$ & $48.4 \mathrm{c} \mathrm{A}$ & $16.2 \mathrm{a} \mathrm{B}$ & $18.0 \mathrm{~b} \mathrm{~A}$ \\
\hline D4-59 x D5-09 & $92 \mathrm{~b} \quad \mathrm{~B}$ & $103 \mathrm{~b} \mathrm{~A}$ & $17.4 \mathrm{~b}$ & $\mathrm{~A}$ & $15.4 \mathrm{~b}$ & B & $48.1 \mathrm{c} \mathrm{A}$ & $50.5 \mathrm{~b} \mathrm{~A}$ & $15.2 \mathrm{~b} \mathrm{~A}$ & $15.7 \mathrm{~d} \mathrm{~A}$ \\
\hline D4-59 x D5-41 & $90 \mathrm{~b} \mathrm{~B}$ & $103 \mathrm{~b} \mathrm{~A}$ & $17.1 \mathrm{~b}$ & A & $16.0 \mathrm{a}$ & A & $47.9 \mathrm{c} \mathrm{A}$ & $48.1 \mathrm{c} \mathrm{A}$ & 15.7 a A & $15.8 \mathrm{~d} \mathrm{~A}$ \\
\hline D4-59 x D5-42 & 94 a B & 104 a A & $17.6 \mathrm{~b}$ & $\mathrm{~A}$ & $16.3 \mathrm{a}$ & A & $45.7 \mathrm{c} \quad \mathrm{B}$ & $51.3 \mathrm{~b} \mathrm{~A}$ & $15.0 \mathrm{~b} \mathrm{~A}$ & $13.9 \mathrm{e} \mathrm{A}$ \\
\hline D4-59 x D5-43 & $87 \mathrm{~d} \mathrm{~B}$ & 104 a A & $18.3 \mathrm{a}$ & $\mathrm{A}$ & $16.4 \mathrm{a}$ & B & $49.3 \mathrm{~b} \quad \mathrm{~A}$ & $49.0 \mathrm{~b} \mathrm{~A}$ & $15.1 \mathrm{~b} \mathrm{~B}$ & $16.8 \mathrm{c} \mathrm{A}$ \\
\hline D4-59 x D5-46 & $92 \mathrm{~b} \quad \mathrm{~B}$ & 106 a A & $17.6 \mathrm{~b}$ & $\mathrm{~A}$ & $15.9 \mathrm{a}$ & B & $49.1 \mathrm{c} \mathrm{A}$ & $48.2 \mathrm{c} \mathrm{A}$ & 15.9 a A & $15.7 \mathrm{~d} A$ \\
\hline D4-59 x D5-48 & 96 a $\mathrm{B}$ & 106 a A & $16.9 \mathrm{~b}$ & $\mathrm{~A}$ & $15.0 \mathrm{~b}$ & $\mathrm{~B}$ & $44.5 \mathrm{c} \quad \mathrm{B}$ & $48.8 \mathrm{c} \mathrm{A}$ & $14.8 \mathrm{~b} \mathrm{~B}$ & $18.3 \mathrm{~b} \mathrm{~A}$ \\
\hline D4-59 x D5-53 & 89 c B & 105 a A & $16.1 \mathrm{~b}$ & $\mathrm{~A}$ & $16.5 \mathrm{a}$ & A & $48.1 \mathrm{c} \mathrm{A}$ & $50.0 \mathrm{~b} \mathrm{~A}$ & 16.7 a A & $14.3 \mathrm{e} \mathrm{B}$ \\
\hline D4-59 x D5-55 & $92 \mathrm{~b} \quad \mathrm{~B}$ & 106 a A & $17.4 \mathrm{~b}$ & $\mathrm{~A}$ & $15.0 \mathrm{~b}$ & $\mathrm{~B}$ & $49.4 \mathrm{~b} \mathrm{~A}$ & $46.9 \mathrm{c} \mathrm{A}$ & $15.2 \mathrm{~b} \quad \mathrm{~B}$ & $18.8 \mathrm{a} \quad \mathrm{A}$ \\
\hline Tropical Plus & $83 \mathrm{~d} \mathrm{~B}$ & $99 \mathrm{~b} \quad \mathrm{~A}$ & $19.6 \mathrm{a}$ & $\mathrm{A}$ & $15.4 \mathrm{~b}$ & $\mathrm{~B}$ & $48.1 \mathrm{c} \mathrm{A}$ & $48.4 \mathrm{c} \mathrm{A}$ & $17.1 \mathrm{a} \mathrm{A}$ & $17.9 \mathrm{~b} \quad \mathrm{~A}$ \\
\hline BRS Vivi & $91 \mathrm{~b} \quad \mathrm{~B}$ & $103 \mathrm{~b} \quad \mathrm{~A}$ & $18.1 \mathrm{a}$ & $\mathrm{A}$ & $16.5 \mathrm{a}$ & $\mathrm{B}$ & $54.5 \mathrm{a} \mathrm{A}$ & $49.1 \mathrm{~b} \mathrm{~A}$ & $16.1 \mathrm{a} \mathrm{A}$ & $12.1 \mathrm{e} \mathrm{B}$ \\
\hline CV (\%) & 2.52 & 1.70 & 4.18 & & 5.60 & & 4.24 & 4.29 & 7.82 & 4.01 \\
\hline Hybrid's average & $90 \quad \mathrm{~B}$ & $104 \mathrm{~A}$ & 18.11 & A & 16.1 & $\mathrm{~B}$ & $48.7 \quad \mathrm{~A}$ & $48.66 \mathrm{~A}$ & $15.3 \quad \mathrm{~B}$ & 16.5 \\
\hline Check's average & 87 & 101 & 18.88 & A & 15.9 & $\mathrm{~B}$ & 51.4 & 48.77 & 16.6 & 14.5 \\
\hline
\end{tabular}

Genotypes followed by the same lowercase letters in the columns or by the same uppercase letters in the lines belong to the same group by the Scott-Knott test at $5 \%$ probability.

studies (Solomon et al., 2011; Santos et al., 2014).

One of the most important traits for the processing of super sweet corn is the ear length (EL), so that ears smaller than $15 \mathrm{~cm}$ are discarded, decreasing the yield (Santos et al., 2014). The means of EL of experimental hybrids ranged from 16.1 to $19.9 \mathrm{~cm}$ in the first season and from 14.7 to $18.2 \mathrm{~cm}$ in the second season; all the values are within desirable range for industrial processing (Table 1). Hybrids D3-20 x D5-46, D3$30 \times$ D5-46 and D2-74 x D5-09 stood out with higher values for EL (Table 1).

The means of the ear diameter (ED) of the experimental hybrids ranged from $44.4 \mathrm{~mm}$ to $54.9 \mathrm{~mm}$ (Table 1 ). In the first sowing season, the experimental hybrids D3-30 x D5-46, D3-30 x D509 and D4-20 x D5-41 and the check BRS Vivi showed the highest estimates for ED, surpassing other genotypes (Table 1).

The wide range of SS values showed that the means of hybrids ranged from $12.8^{\circ}$ Brix (D2-74 x D5-09) to $17.2^{\circ}$ Brix (D3-30 x D5-46) in the first season (Table 1), whose values corroborate other studies, showing values between $11.2^{\circ}$ Brix to $17^{\circ}$ Brix (Mamede et al., 2014; Perfeito et al., 2017; Machado Filho et al., 2018).

The experimental hybrids D3-30 x D5-46, D3-30 x D5-42 and D3-39 x D555 , along with the check Tropical Plus, showed the highest values of soluble solids (SS) (Table 1), being considered promising (Machado Filho et al., 2018), once that super sweet genotypes shall present SS above $15^{\circ}$ Brix (Pereira Filho et al., 2016).

Promising results were also obtained for UEY, in which only the experimental hybrid D4-20 x D5-55 obtained mean below $12.0 \mathrm{t} \mathrm{ha}^{-1}$ in the first growing season, which according to Pereira Filho et al. (2016) is the lowest value recommended for a profitable crop. The experimental hybrids with the highest means of UEY showed high potential in comparison to check hybrids and the experimental hybrid D3-20 x D541 (25.7 $\left.\mathrm{t} \mathrm{ha}^{-1}\right)$ had the highest mean together with other four experimental hybrids (Table 2).

In the second sowing season, most experimental hybrids showed inferior performance for UEY due to the environmental effects resulting from variations of rainfall and daily minimum and maximum temperatures in Guarapuava. Better rainfall distribution during flowering and grain filling were observed in the first season. In the second season an increase in the average temperature and a reduction in the average of the daily thermal amplitude was observed in Guarapuava (Shioga et al., 2017). UEY values ranged from 9.1 $\mathrm{t} \mathrm{ha}^{-1}$ (D3-33 x D5-42) to $20.5 \mathrm{t} \mathrm{ha}^{-1}$ (D2$17 \times$ D5-46), and only nine experimental hybrids surpassed the average of the checks (Table 2).

In the first sowing season only five hybrids showed HEY superior than the other experimental hybrids and checks, whose values surpassed $16.0 \mathrm{t} \mathrm{ha}^{-1}$. In the second sowing season the average of HEY decreased considerably, and four hybrids were superior comparing to the others, whose values exceeded $13.5 \mathrm{t}$ ha $^{-1}$. The hybrid D3-30 x D5-46 (Table 2) stood out due to its high performance in both seasons.

In the first sowing season, nine hybrids formed the superior group, whose values of GY were above $8.3 \mathrm{t}$ $\mathrm{ha}^{-1}$, surpassing the checks. In the second season, 37 hybrids did not differ from 
Table 2. Means of unhusked ear yield (UEY), husked ear yield (HEY) and grain yield (GY) of super sweet corn experimental hybrids and checks evaluated in two sowing seasons in 2016/2017 in Guarapuava-PR. Guarapuava, UNICENTRO, 2021.

\begin{tabular}{|c|c|c|c|c|c|c|c|c|c|c|c|c|c|c|c|c|c|c|}
\hline \multirow{3}{*}{$\begin{array}{l}\text { Genotype } \\
\text { D2-17 x D5-09 }\end{array}$} & \multicolumn{6}{|c|}{ UEY $\left(\mathrm{t} \mathrm{ha}^{-1}\right)$} & \multicolumn{6}{|c|}{ HEY $\left(\right.$ t ha $\left.^{-1}\right)$} & \multicolumn{6}{|c|}{ GY $\left(\mathrm{t} \mathrm{ha}^{-1}\right)$} \\
\hline & \multicolumn{3}{|c|}{ Season 1} & \multicolumn{3}{|c|}{ Season 2} & \multicolumn{3}{|c|}{ Season 1} & \multicolumn{3}{|c|}{ Season 2} & \multicolumn{3}{|c|}{ Season 1} & \multicolumn{3}{|c|}{ Season 2} \\
\hline & 21.6 & $\mathrm{~b}$ & $\mathrm{~A}$ & 11.6 & $\mathrm{~d}$ & $\mathrm{~B}$ & 14.4 & $\mathrm{~b}$ & $\mathrm{~A}$ & 8.1 & $\mathrm{~b}$ & $\mathrm{~B}$ & 6.5 & $\mathrm{c}$ & $\mathrm{A}$ & 4.7 & $\mathrm{~b}$ & $\mathrm{~B}$ \\
\hline D2-17 x D5-41 & 14.6 & e & A & 15.2 & $\mathrm{~b}$ & A & 10.4 & $\mathrm{~b}$ & A & 10.7 & $\mathrm{~b}$ & A & 5.3 & $\mathrm{~d}$ & A & 6.3 & $\mathrm{a}$ & A \\
\hline D2-17 x D5-42 & 18.7 & $\mathrm{c}$ & A & 13.4 & $\mathrm{c}$ & $\mathrm{B}$ & 12.1 & $\mathrm{~b}$ & A & 9.8 & $\mathrm{~b}$ & A & 7.4 & $\mathrm{~b}$ & A & 5.8 & $\mathrm{~b}$ & B \\
\hline D2-17 x D5-43 & 22.2 & $\mathrm{~b}$ & A & 15.5 & $b$ & $\mathrm{~B}$ & 15.6 & $\mathrm{~b}$ & A & 10.3 & $\mathrm{~b}$ & A & 7.2 & $\mathrm{~b}$ & A & 5.7 & $\mathrm{~b}$ & A \\
\hline D2-17 x D5-46 & 18.7 & $\mathrm{c}$ & A & 20.5 & $\mathrm{a}$ & A & 14.1 & $\mathrm{~b}$ & A & 13.6 & $\mathrm{a}$ & A & 7.9 & $\mathrm{~b}$ & A & 7.0 & $\mathrm{a}$ & A \\
\hline D2-17 x D5-48 & 14.2 & e & A & 15.6 & $\mathrm{~b}$ & $\mathrm{~A}$ & 10.1 & $\mathrm{~b}$ & A & 11.5 & $\mathrm{~b}$ & A & 4.9 & $\mathrm{~d}$ & $\mathrm{~B}$ & 7.2 & $\mathrm{a}$ & A \\
\hline D2-17 x D5-53 & 14.7 & e & $\mathrm{A}$ & 16.6 & $\mathrm{~b}$ & $\mathrm{~A}$ & 9.3 & $\mathrm{c}$ & A & 11.9 & $\mathrm{~b}$ & $\mathrm{~A}$ & 4.1 & $\mathrm{~d}$ & B & 7.3 & $\mathrm{a}$ & A \\
\hline D2-17 x D5-55 & 20.1 & $\mathrm{c}$ & $\mathrm{A}$ & 14.9 & $\mathrm{~b}$ & B & 13.7 & $\mathrm{~b}$ & A & 11.0 & $\mathrm{~b}$ & A & 8.4 & $\mathrm{a}$ & A & 6.7 & $\mathrm{a}$ & B \\
\hline D2-74 x D5-09 & 17.4 & $\mathrm{~d}$ & A & 13.4 & $\mathrm{c}$ & B & 11.7 & $\mathrm{~b}$ & A & 8.9 & $\mathrm{~b}$ & A & 5.3 & $\mathrm{~d}$ & A & 5.3 & $\mathrm{~b}$ & A \\
\hline D2-74 x D5-41 & 12.8 & e & B & 17.8 & $\mathrm{a}$ & A & 8.5 & $\mathrm{c}$ & A & 11.7 & $\mathrm{~b}$ & A & 4.4 & $\mathrm{~d}$ & B & 7.0 & $\mathrm{a}$ & A \\
\hline D2-74 x D5-42 & 18.5 & $\mathrm{c}$ & A & 13.6 & $\mathrm{c}$ & B & 12.4 & $\mathrm{~b}$ & A & 10.0 & $\mathrm{~b}$ & A & 5.9 & $\mathrm{c}$ & A & 5.5 & $\mathrm{~b}$ & A \\
\hline D2-74 x D5-43 & 18.1 & $\mathrm{c}$ & A & 13.3 & $\mathrm{c}$ & B & 12.2 & $\mathrm{~b}$ & A & 9.4 & $\mathrm{~b}$ & A & 5.9 & $\mathrm{c}$ & A & 4.5 & $\mathrm{~b}$ & A \\
\hline D2-74 x D5-46 & 16.9 & $\mathrm{~d}$ & A & 18.3 & $\mathrm{a}$ & A & 11.8 & $\mathrm{~b}$ & A & 12.4 & $\mathrm{~b}$ & A & 6.2 & $\mathrm{c}$ & A & 6.5 & $\mathrm{a}$ & A \\
\hline D2-74 x D5-48 & 20.2 & $\mathrm{c}$ & A & 13.9 & $\mathrm{c}$ & B & 13.5 & $\mathrm{~b}$ & A & 9.5 & $\mathrm{~b}$ & A & 7.9 & $\mathrm{~b}$ & A & 6.2 & $\mathrm{a}$ & B \\
\hline D2-74 x D5-53 & 12.9 & e & B & 17.3 & $\mathrm{~b}$ & A & 8.7 & $\mathrm{c}$ & A & 11.9 & $\mathrm{~b}$ & A & 3.9 & $\mathrm{~d}$ & $\mathrm{~B}$ & 7.2 & $\mathrm{a}$ & A \\
\hline D2-74 x D5-55 & 14.0 & e & A & 14.8 & $\mathrm{~b}$ & A & 9.9 & $\mathrm{c}$ & A & 10.4 & $\mathrm{~b}$ & A & 5.0 & $\mathrm{~d}$ & A & 6.0 & $\mathrm{a}$ & A \\
\hline D3-20 x D5-09 & 18.0 & $\mathrm{c}$ & A & 10.0 & $\mathrm{~d}$ & B & 11.5 & $\mathrm{~b}$ & A & 7.4 & $\mathrm{c}$ & A & 5.7 & $\mathrm{c}$ & A & 4.9 & $\mathrm{~b}$ & A \\
\hline D3-20 x D5-41 & 25.7 & $\mathrm{a}$ & A & 12.2 & $\mathrm{c}$ & $\mathrm{B}$ & 18.1 & $\mathrm{a}$ & A & 8.7 & $\mathrm{~b}$ & $\mathrm{~B}$ & 8.8 & $\mathrm{a}$ & A & 6.2 & $\mathrm{a}$ & B \\
\hline D3-20 x D5-42 & 24.1 & $\mathrm{a}$ & A & 13.2 & $\mathrm{c}$ & B & 15.7 & $\mathrm{~b}$ & A & 9.7 & $\mathrm{~b}$ & B & 8.5 & $\mathrm{a}$ & A & 5.4 & $\mathrm{~b}$ & B \\
\hline D3-20 x D5-43 & 18.4 & $\mathrm{c}$ & A & 12.4 & $\mathrm{c}$ & $\mathrm{B}$ & 11.8 & $\mathrm{~b}$ & A & 8.9 & $\mathrm{~b}$ & A & 6.3 & $\mathrm{c}$ & A & 5.7 & $\mathrm{~b}$ & A \\
\hline D3-20 x D5-46 & 19.6 & $\mathrm{c}$ & A & 16.3 & $\mathrm{~b}$ & B & 13.4 & $\mathrm{~b}$ & A & 10.7 & $\mathrm{~b}$ & A & 7.3 & $\mathrm{~b}$ & A & 6.3 & $\mathrm{a}$ & A \\
\hline D3-20 x D5-48 & 14.5 & $\mathrm{e}$ & A & 12.3 & $\mathrm{c}$ & A & 10.3 & $\mathrm{~b}$ & A & 8.5 & $\mathrm{~b}$ & A & 4.7 & $\mathrm{~d}$ & A & 5.0 & $\mathrm{~b}$ & A \\
\hline D3-20 x D5-53 & 15.6 & $\mathrm{~d}$ & A & 15.5 & $\mathrm{~b}$ & A & 10.1 & $\mathrm{~b}$ & $\mathrm{~A}$ & 10.8 & $\mathrm{~b}$ & A & 5.0 & $\mathrm{~d}$ & $\mathrm{~B}$ & 6.8 & $\mathrm{a}$ & A \\
\hline D3-20 x D5-55 & 25.4 & $\mathrm{a}$ & $\mathrm{A}$ & 16.7 & $\mathrm{~b}$ & B & 16.0 & $\mathrm{a}$ & $\mathrm{A}$ & 11.4 & $\mathrm{~b}$ & A & 8.1 & $\mathrm{~b}$ & $\mathrm{~A}$ & 6.8 & $\mathrm{a}$ & $\mathrm{A}$ \\
\hline D3-30 x D5-09 & 20.2 & $\mathrm{c}$ & $\mathrm{A}$ & 11.1 & $\mathrm{~d}$ & $\mathrm{~B}$ & 14.9 & $\mathrm{~b}$ & $\mathrm{~A}$ & 7.9 & $\mathrm{c}$ & B & 7.4 & $\mathrm{~b}$ & $\mathrm{~A}$ & 5.1 & $\mathrm{~b}$ & B \\
\hline D3-30 x D5-41 & 18.3 & $\mathrm{c}$ & A & 9.5 & $\mathrm{~d}$ & B & 13.3 & $\mathrm{~b}$ & $\mathrm{~B}$ & 13.6 & $\mathrm{a}$ & A & 7.1 & $\mathrm{~b}$ & $\mathrm{~A}$ & 4.6 & $\mathrm{~b}$ & B \\
\hline D3-30 x D5-42 & 16.6 & $\mathrm{~d}$ & A & 15.1 & $\mathrm{~b}$ & $\mathrm{~A}$ & 12.0 & $\mathrm{~b}$ & $\mathrm{~A}$ & 10.4 & $\mathrm{~b}$ & A & 6.2 & $\mathrm{c}$ & A & 5.1 & $\mathrm{~b}$ & A \\
\hline D3-30 x D5-43 & 21.1 & c & A & 12.2 & $\mathrm{c}$ & B & 14.1 & $\mathrm{~b}$ & $\mathrm{~A}$ & 9.1 & $\mathrm{~b}$ & A & 6.5 & $\mathrm{c}$ & A & 5.5 & $\mathrm{~b}$ & $\mathrm{~A}$ \\
\hline D3-30 x D5-46 & 23.1 & $\mathrm{~b}$ & A & 19.3 & $\mathrm{a}$ & B & 16.5 & $\mathrm{a}$ & A & 13.6 & $\mathrm{a}$ & A & 8.1 & $\mathrm{~b}$ & $\mathrm{~A}$ & 7.8 & $\mathrm{a}$ & $\mathrm{A}$ \\
\hline D3-30 x D5-48 & 18.2 & $\mathrm{c}$ & $\mathrm{A}$ & 15.1 & $\mathrm{~b}$ & $\mathrm{~B}$ & 12.5 & $\mathrm{~b}$ & A & 10.9 & $\mathrm{~b}$ & A & 6.4 & $\mathrm{c}$ & A & 6.5 & $\mathrm{a}$ & $\mathrm{A}$ \\
\hline D3-30 x D5-53 & 15.7 & d & A & 13.5 & $\mathrm{c}$ & A & 10.5 & $\mathrm{~b}$ & A & 9.7 & $\mathrm{~b}$ & A & 4.6 & $\mathrm{~d}$ & B & 6.3 & $\mathrm{a}$ & A \\
\hline D3-30 x D5-55 & 16.3 & d & A & 14.9 & $\mathrm{~b}$ & A & 10.3 & $\mathrm{~b}$ & A & 10.4 & $\mathrm{~b}$ & A & 4.8 & $\mathrm{~d}$ & $\mathrm{~B}$ & 6.6 & $\mathrm{a}$ & A \\
\hline D3-33 x D5-09 & 16.9 & d & $\mathrm{A}$ & 10.5 & $\mathrm{~d}$ & B & 12.5 & $\mathrm{~b}$ & A & 7.0 & $\mathrm{c}$ & A & 6.3 & $\mathrm{c}$ & A & 4.5 & $\mathrm{~b}$ & B \\
\hline D3-33 x D5-41 & 20.5 & $\mathrm{c}$ & A & 12.2 & $\mathrm{c}$ & $\mathrm{B}$ & 13.9 & $\mathrm{~b}$ & A & 9.1 & $\mathrm{~b}$ & A & 6.9 & $\mathrm{~b}$ & A & 6.0 & $\mathrm{a}$ & A \\
\hline D3-33 x D5-42 & 19.2 & $\mathrm{c}$ & A & 9.1 & d & $\mathrm{B}$ & 12.7 & $\mathrm{~b}$ & A & 7.1 & $\mathrm{c}$ & A & 6.1 & $\mathrm{c}$ & A & 4.7 & $\mathrm{~b}$ & A \\
\hline D3-33 x D5-43 & 16.3 & $\mathrm{~d}$ & A & 10.7 & $\mathrm{~d}$ & $\mathrm{~B}$ & 10.7 & $\mathrm{~b}$ & A & 6.9 & $\mathrm{c}$ & A & 5.3 & $\mathrm{~d}$ & A & 4.4 & $\mathrm{~b}$ & A \\
\hline D3-33 x D5-46 & 20.8 & $\mathrm{c}$ & A & 17.0 & $\mathrm{~b}$ & $\mathrm{~B}$ & 14.1 & $\mathrm{~b}$ & A & 11.9 & $\mathrm{~b}$ & A & 7.1 & $\mathrm{~b}$ & A & 6.4 & $\mathrm{a}$ & A \\
\hline D3-33 x D5-48 & 21.1 & $\mathrm{c}$ & A & 16.2 & $\mathrm{~b}$ & B & 14.3 & $\mathrm{~b}$ & A & 10.6 & $\mathrm{~b}$ & A & 8.5 & $\mathrm{a}$ & A & 6.3 & $\mathrm{a}$ & B \\
\hline D3-33 x D5-53 & 16.9 & $\mathrm{~d}$ & A & 15.9 & $\mathrm{~b}$ & A & 11.3 & $\mathrm{~b}$ & A & 11.1 & $\mathrm{~b}$ & A & 5.6 & $\mathrm{c}$ & A & 6.4 & $\mathrm{a}$ & A \\
\hline D3-33 x D5-55 & 12.3 & e & B & 15.1 & $\mathrm{~b}$ & A & 8.4 & $\mathrm{c}$ & A & 10.8 & $\mathrm{~b}$ & A & 4.4 & $\mathrm{~d}$ & $\mathrm{~B}$ & 6.9 & $\mathrm{a}$ & A \\
\hline D3-39 x D5-09 & 18.7 & $\mathrm{c}$ & A & 11.4 & $\mathrm{~d}$ & B & 12.3 & $\mathrm{~b}$ & A & 7.5 & $\mathrm{c}$ & A & 6.6 & $\mathrm{c}$ & A & 5.1 & $\mathrm{~b}$ & A \\
\hline D3-39 x D5-41 & 19.4 & $\mathrm{c}$ & A & 18.2 & $\mathrm{a}$ & A & 13.4 & $\mathrm{~b}$ & A & 11.5 & $\mathrm{~b}$ & A & 6.3 & $\mathrm{c}$ & A & 6.5 & $\mathrm{a}$ & A \\
\hline D3-39 x D5-42 & 23.4 & $\mathrm{~b}$ & A & 13.1 & $\mathrm{c}$ & B & 17.1 & $\mathrm{a}$ & A & 8.6 & $\mathrm{~b}$ & B & 9.3 & $\mathrm{a}$ & A & 5.7 & $\mathrm{~b}$ & B \\
\hline D3-39 x D5-43 & 18.7 & $\mathrm{c}$ & A & 17.2 & $\mathrm{~b}$ & A & 13.8 & $\mathrm{~b}$ & A & 12.8 & $\mathrm{~b}$ & A & 7.9 & $\mathrm{~b}$ & A & 8.5 & $\mathrm{a}$ & A \\
\hline D3-39 x D5-46 & 19.4 & $\mathrm{c}$ & A & 14.7 & $\mathrm{~b}$ & B & 14.1 & $\mathrm{~b}$ & A & 9.5 & $\mathrm{~b}$ & A & 6.7 & $\mathrm{c}$ & $\mathrm{A}$ & 5.3 & $\mathrm{~b}$ & A \\
\hline D3-39 x D5-48 & 24.9 & $\mathrm{a}$ & A & 10.6 & $\mathrm{~d}$ & B & 18.1 & $\mathrm{a}$ & A & 7.6 & $\mathrm{c}$ & B & 10.0 & $\mathrm{a}$ & A & 4.9 & $\mathrm{~b}$ & B \\
\hline D3-39 x D5-53 & 14.3 & $\mathrm{e}$ & B & 18.9 & $\mathrm{a}$ & A & 9.5 & $\mathrm{c}$ & A & 12.5 & $\mathrm{~b}$ & A & 4.4 & $\mathrm{~d}$ & $\mathrm{~B}$ & 7.1 & $\mathrm{a}$ & A \\
\hline D3-39 x D5-55 & 15.7 & $\mathrm{~d}$ & A & 14.8 & $\mathrm{~b}$ & A & 10.9 & $\mathrm{~b}$ & A & 10.0 & $\mathrm{~b}$ & A & 5.9 & $\mathrm{c}$ & A & 5.8 & $\mathrm{~b}$ & A \\
\hline D4-20 x D5-09 & 17.1 & $\mathrm{~d}$ & A & 13.7 & $\mathrm{c}$ & B & 11.9 & $\mathrm{~b}$ & $\mathrm{~A}$ & 10.2 & $\mathrm{~b}$ & A & 5.9 & $\mathrm{c}$ & A & 6.8 & $\mathrm{a}$ & A \\
\hline D4-20 x D5-41 & 19.9 & $\mathrm{c}$ & A & 13.6 & $\mathrm{c}$ & B & 14.9 & $\mathrm{~b}$ & A & 9.5 & $\mathrm{~b}$ & A & 8.9 & $\mathrm{a}$ & A & 5.1 & $\mathrm{~b}$ & B \\
\hline
\end{tabular}


Table 2 continuation

\begin{tabular}{|c|c|c|c|c|c|c|c|c|c|c|c|c|c|c|c|c|c|}
\hline \multirow{3}{*}{$\begin{array}{l}\text { Genotype } \\
\text { D4-20 x D5-42 }\end{array}$} & \multicolumn{6}{|c|}{$\operatorname{UEY}\left(\mathrm{t} \mathrm{ha}^{-1}\right)$} & \multicolumn{5}{|c|}{ HEY (t ha-1) } & \multicolumn{6}{|c|}{ GY $\left(\mathrm{t} \mathrm{ha}^{-1}\right)$} \\
\hline & \multicolumn{3}{|c|}{ Season 1} & \multicolumn{3}{|c|}{ Season 2} & \multicolumn{2}{|c|}{ Season 1} & \multicolumn{3}{|c|}{ Season 2} & \multicolumn{3}{|c|}{ Season 1} & \multicolumn{3}{|c|}{ Season 2} \\
\hline & 21.7 & $\mathrm{~b}$ & $\mathrm{~A}$ & 14.7 & $\mathrm{~b}$ & B & 15.0 & b A & 9.6 & $\mathrm{~b}$ & $\mathrm{~A}$ & 8.6 & $\mathrm{a}$ & $\mathrm{A}$ & 5.9 & $\mathrm{~b}$ & B \\
\hline D4-20 x D5-43 & 18.4 & $\mathrm{c}$ & A & 12.4 & $\mathrm{c}$ & B & 13.4 & b A & 9.5 & b & A & 7.6 & $\mathrm{~b}$ & A & 6.2 & $\mathrm{a}$ & A \\
\hline D4-20 x D5-46 & 20.1 & $\mathrm{c}$ & A & 16.8 & $\mathrm{~b}$ & B & 14.1 & $\mathrm{~b}$ & 11.4 & b & A & 7.2 & $\mathrm{~b}$ & A & 6.5 & $\mathrm{a}$ & A \\
\hline D4-20 x D5-48 & 18.6 & $\mathrm{c}$ & A & 14.8 & $b$ & B & 12.1 & $\mathrm{~b}$ & 10.3 & $\mathrm{~b}$ & A & 6.0 & $\mathrm{c}$ & A & 6.3 & $\mathrm{a}$ & A \\
\hline D4-20 x D5-53 & 13.1 & e & A & 13.7 & $\mathrm{c}$ & A & 9.2 & A & 9.6 & $\mathrm{~b}$ & A & 4.6 & d & B & 6.2 & $\mathrm{a}$ & A \\
\hline D4-20 x D5-55 & 11.6 & $\mathrm{e}$ & B & 19.9 & $\mathrm{a}$ & A & 8.1 & A & 11.3 & $\mathrm{~b}$ & A & 4.7 & d & A & 3.9 & $\mathrm{~b}$ & A \\
\hline D4-59 x D5-09 & 21.6 & $\mathrm{~b}$ & A & 12.3 & $\mathrm{c}$ & B & 14.7 & A & 8.8 & $\mathrm{~b}$ & B & 8.3 & $\mathrm{a}$ & A & 6.4 & $\mathrm{a}$ & B \\
\hline D4-59 x D5-41 & 22.7 & $\mathrm{~b}$ & A & 19.9 & $\mathrm{a}$ & B & 14.4 & A & 14.4 & $\mathrm{a}$ & A & 6.4 & $\mathrm{c}$ & A & 7.2 & $\mathrm{a}$ & A \\
\hline D4-59 x D5-42 & 16.6 & d & A & 13.9 & $\mathrm{c}$ & A & 10.3 & A & 9.7 & $\mathrm{~b}$ & A & 6.2 & $\mathrm{c}$ & A & 5.6 & $\mathrm{~b}$ & A \\
\hline D4-59 x D5-43 & 21.7 & $\mathrm{~b}$ & A & 13.9 & $\mathrm{c}$ & B & 14.8 & A & 10.2 & $\mathrm{~b}$ & A & 7.4 & $\mathrm{~b}$ & A & 5.6 & $\mathrm{~b}$ & B \\
\hline D4-59 x D5-46 & 18.7 & $\mathrm{c}$ & A & 16.4 & $\mathrm{~b}$ & A & 13.3 & A & 11.5 & $\mathrm{~b}$ & A & 6.6 & $\mathrm{c}$ & A & 6.6 & $\mathrm{a}$ & A \\
\hline D4-59 x D5-48 & 19.4 & $\mathrm{c}$ & A & 19.4 & $\mathrm{a}$ & A & 12.4 & A & 12.9 & $\mathrm{~b}$ & A & 7.1 & $\mathrm{~b}$ & A & 7.3 & $\mathrm{a}$ & A \\
\hline D4-59 x D5-53 & 18.7 & $\mathrm{c}$ & A & 15.6 & $\mathrm{~b}$ & B & 12.3 & A & 10.9 & $\mathrm{~b}$ & A & 6.2 & $\mathrm{c}$ & A & 6.3 & $\mathrm{a}$ & A \\
\hline D4-59 x D5-55 & 19.0 & $\mathrm{c}$ & A & 16.4 & $\mathrm{~b}$ & A & $12 . .8$ & A & 11.5 & $\mathrm{~b}$ & A & 6.5 & $\mathrm{c}$ & A & 6.8 & $\mathrm{a}$ & A \\
\hline Tropical Plus & 16.3 & d & A & 16.4 & $\mathrm{~b}$ & A & 11.8 & A & 12.1 & $\mathrm{~b}$ & A & 6.1 & $\mathrm{c}$ & A & 6.6 & $\mathrm{a}$ & A \\
\hline BRS Vivi & 14.9 & $\mathrm{e}$ & A & 17.3 & $\mathrm{~b}$ & A & 10.5 & A & 11.9 & $\mathrm{~b}$ & A & 5.8 & $\mathrm{c}$ & A & 6.7 & $\mathrm{a}$ & A \\
\hline CV $(\%)$ & 10. & & & 10.10 & & & 11.66 & & 14.87 & & & 15.6 & & & 13.2 & & \\
\hline Hybrid's average & 18. & 5 & & 14.7 & B & & 12.7 & A & 10.3 & $\mathrm{~B}$ & & 6.5 & A & & 6.0 & A & \\
\hline Check's average & 15. & & A & 16.9 & A & & 11.2 & A & 12.0 & A & & 5.9 & A & & 6.7 & A & \\
\hline
\end{tabular}

Genotypes followed by the same lowercase letters in the columns or by the same uppercase letters in the lines belong to the same group by the Scott-Knott test at 5\% probability. First season= sowing performed on October 15, 2016; second season= sowing performed on December 17, 2016.

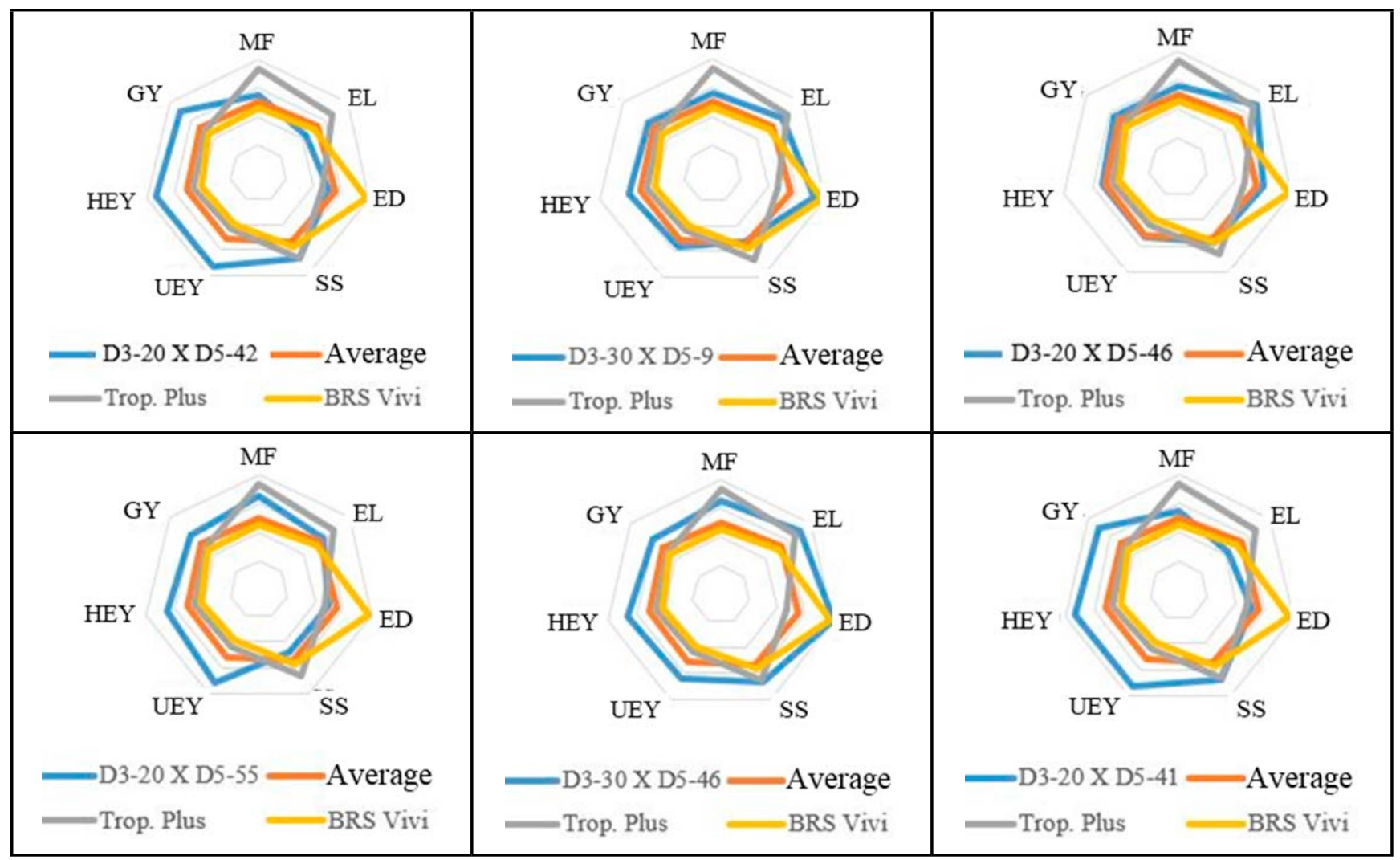

Figure 1. Experimental hybrids selected based on the values of $\mathrm{Z}$ index $(I z)$ using the standardized means of traits: male flowering (MF), ear length (EL), ear diameter (ED), soluble solid content (SS), unhusked ear yield (UEY), husked ear yield (HEY) and grain yield (GY), evaluated in the first sowing season in 2016/2017, in Guarapuava-PR. First season= sowing performed on October 15, 2016. Guarapuava, UNICENTRO, 2021. 
Table 3. Standardized means of the evaluated traits, values of $Z$ index $(I z)$ and variances $\left(\sigma^{2}\right)$ of 10 hybrids which surpassed the checks in the first and second sowing seasons in 2016/2017 in Guarapuava-PR. Guarapuava, UNICENTRO, 2021.

\begin{tabular}{|c|c|c|c|c|c|c|c|c|c|}
\hline \multirow{2}{*}{ Genotypes } & MF & EL & ED & SS & UEY & HEY & GY & \multirow{2}{*}{$\mathbf{I}_{\mathrm{z}}$} & \multirow{2}{*}{$\sigma^{2}$} \\
\hline & \multicolumn{7}{|c|}{$1^{\text {st }}$ Season } & & \\
\hline D3-30 X D5-46 & 6.48 & 7.06 & 7.98 & 6.74 & 6.45 & 6.68 & 6.11 & 47.50 & 0.31 \\
\hline D3-20 X D5-41 & 5.45 & 6.31 & 5.14 & 4.79 & 7.25 & 7.36 & 6.67 & 42.97 & 1.17 \\
\hline D3-20 X D5-55 & 6.48 & 5.64 & 4.90 & 4.82 & 7.17 & 6.48 & 6.12 & 41.61 & 0.75 \\
\hline D3-30 X D5-09 & 5.58 & 6.20 & 7.20 & 5.20 & 5.57 & 6.00 & 5.63 & 41.37 & 0.37 \\
\hline D3-39 X D5-42 & 4.16 & 5.11 & 5.51 & 5.82 & 6.56 & 6.95 & 7.03 & 41.15 & 1.10 \\
\hline D3-20 X D5-42 & 4.55 & 5.98 & 4.77 & 6.12 & 6.76 & 6.34 & 6.45 & 40.97 & 0.69 \\
\hline D3-39 X D5-48 & 2.87 & 5.60 & 4.92 & 5.56 & 7.00 & 7.39 & 7.54 & 40.87 & 2.36 \\
\hline D4-20 X D5-46 & 6.74 & 6.24 & 5.48 & 5.53 & 5.52 & 5.63 & 5.46 & 40.61 & 0.21 \\
\hline D3-20 X D5-46 & 5.58 & 6.95 & 6.02 & 5.74 & 5.35 & 5.32 & 5.60 & 40.57 & 0.28 \\
\hline D4-20 X D5-41 & 4.81 & 4.40 & 6.64 & 6.51 & 5.46 & 6.00 & 6.72 & 40.53 & 0.74 \\
\hline Tropical Plus & 7.39 & 6.65 & 4.69 & 6.62 & 4.34 & 4.64 & 4.74 & 39.08 & 1.42 \\
\hline BRS Vivi & 4.55 & 5.04 & 7.79 & 5.71 & 3.93 & 4.09 & 4.49 & 35.60 & 1.64 \\
\hline \multicolumn{10}{|c|}{$2^{\text {nd }}$ Season } \\
\hline D2-17 X D5-46 & 5.98 & 4.97 & 4.82 & 6.87 & 7.15 & 6.91 & 6.07 & 42.75 & 0.85 \\
\hline D3-30 X D5-46 & 4.81 & 6.38 & 5.30 & 5.33 & 6.72 & 6.89 & 6.92 & 42.34 & 0.71 \\
\hline D3-20 X D5-55 & 5.20 & 5.91 & 7.91 & 5.13 & 5.75 & 5.61 & 5.82 & 41.33 & 0.81 \\
\hline D2-74 X D5-41 & 6.17 & 6.54 & 5.57 & 4.65 & 6.16 & 5.78 & 6.09 & 40.96 & 0.35 \\
\hline D4-59 X D5-41 & 5.40 & 4.97 & 4.61 & 4.71 & 6.92 & 7.37 & 6.26 & 40.24 & 1.07 \\
\hline D3-20 X D5-46 & 6.17 & 6.07 & 6.42 & 5.29 & 5.59 & 5.23 & 5.30 & 40.08 & 0.20 \\
\hline D3-39 X D5-53 & 5.01 & 5.33 & 4.84 & 5.37 & 6.56 & 6.27 & 6.10 & 39.49 & 0.41 \\
\hline D3-39 X D5-43 & 5.59 & 5.39 & 5.98 & 1.82 & 5.94 & 6.45 & 7.73 & 38.90 & 2.91 \\
\hline D4-20 X D5-09 & 5.78 & 5.23 & 5.89 & 6.57 & 4.62 & 4.91 & 5.82 & 38.82 & 0.46 \\
\hline D2-74 X D5-46 & 5.20 & 5.59 & 4.84 & 4.42 & 6.34 & 6.22 & 5.50 & 38.12 & 0.51 \\
\hline BRS Vivi & 6.95 & 5.70 & 5.27 & 2.36 & 5.97 & 5.95 & 5.74 & 37.95 & 1.82 \\
\hline Tropical Plus & 5.20 & 4.02 & 4.86 & 5.73 & 5.62 & 6.00 & 5.61 & 37.04 & 0.41 \\
\hline
\end{tabular}

First season= sowing performed on October 15, 2016; second season= sowing performed on December 17, 2016.

the checks (Table 2). The hybrids D2-17 x D5-55, D3-20 x D5-41, D3-33 x D548 and D4-59 x D5-09 were classified in the group which showed the highest means of GY in both seasons (Table 2).

Considering both seasons, 16 experimental hybrids showed superior means of UEY, HEY and GY, or did not differ from the checks (Table 2), forming the group of the most promising genotypes, with high yield values. For these mentioned traits, the first season was the best one for genotypes expressing their genetics potential, in which the means of the experimental hybrids D3-30 x D5-46, D3-20 x D5-55, D3-20 x D5-41, D3-39 x D5-42, D3-39 $x$ D5-48 were superior to both checks
(Table 2).

UEY, HEY and GY are determinant for the most promising hybrids, even though $\mathrm{CE}$ and DE are directly related to productivity (Solomon et al., 2011) as well as SS are determinant for the quality of the super sweet corn (Santos et al., 2014; Pereira Filho et al., 2016). About $90 \%$ of super sweet corn production is destined to the processing industry, requiring proper and uniform size of ears and grain quality, contributing for a good industrial yield (Luz et al., 2014).

$\mathrm{Z}$ index $\left(I_{Z}\right)$ allows the use of standardized data of the traits so that they can be considered together in the selection, without the need to obtain estimates of genetic's and phenotypic's covariances (Ramalho et al., 2012). Analysis of variance of $I_{Z}$ estimates showed significant effect both for hybrids and for hybrids $\mathrm{x}$ seasons interaction $(\mathrm{p}<0.05)$. Considering the $I z$ values of both sowing seasons, hybrids with selection potential were noticed, considering the evaluated traits (Table 3).

The hybrids D3-30 x D5-46, D3-20 x D5-46 and D3-20 x D5-55 were among the ten ones which showed estimates of Iz superior to the checks in both sowing seasons (Table 3). Besides these hybrids, in the first sowing season, the hybrids D3-20 x D5-42, D3-30 x D5-09 and D3-20 x D5-41 stood out, with high $I z$ estimates (Figure 1). In the second 


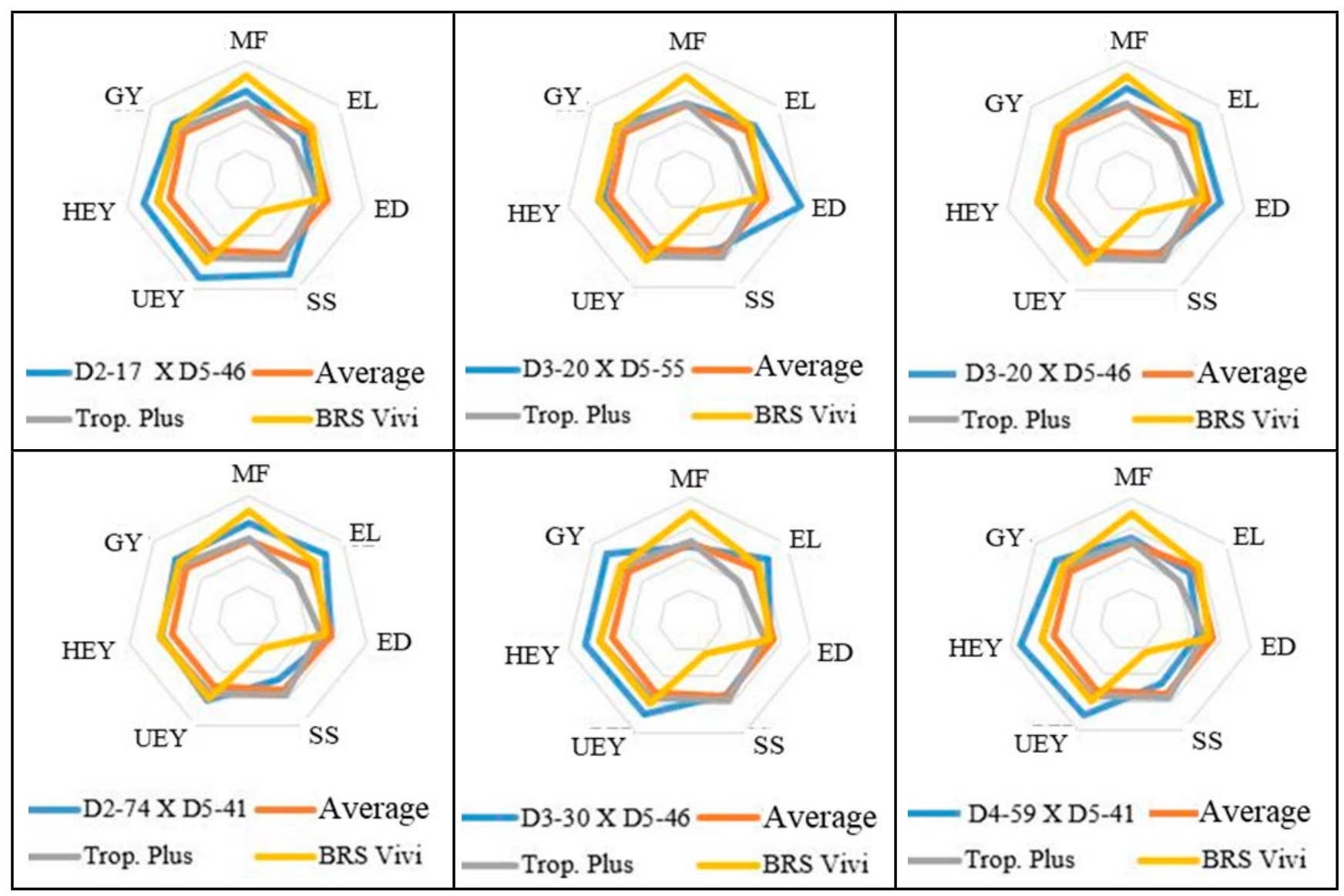

Figure 2. Experimental hybrids selected based on the values of $Z$ index ( $I z)$ using the standardized means of traits: male flowering (MF), ear length (EL), ear diameter (ED), soluble solid content (SS), unhusked ear yield (UEY), husked ear yield (HEY) and grain yield (GY), evaluated in the second sowing season in 2016/2017 in Guarapuava-PR. Second season= sowing performed on December $17,2016$. Guarapuava, UNICENTRO, 2021.

season, the hybrids D2-17 x D5-46, D2-74 x D5-41 and D4-59 x D5-41stood out (Figure 2).

The analysis of the contribution of each trait for the $I_{Z}$ index allows to obtain information on the phenotypic performance of the evaluated genotypes (Wu et al., 2015). Evaluating the promising hybrids in the first season, based on $I z$ values and in the standardized means of each trait which make up this index (Table 3 ) it was possible to verify that the contribution of UEY, HEY and GY was lower for the hybrid D3-20 $x$ D5-46, which did not stand out in relation to the checks (Figure 1), which limits the selection (Reis et al., 2011), considering that they are traits related to yield.

The hybrid D2-17 x D5-46 showed a high estimate of $I_{z}$ in the second sowing season due to its high standardized means of most of the traits which make up the index, mainly the ones related to yield, surpassing both the general average and the checks (Figure 2), highlighting its productive potential and ear standard suitable for industrial processing (Luz et al., 2014; Perfeito et al., 2017; Machado et al., 2018).

In the second sowing season, based on the estimate of $I_{Z}$ and in the standardized values of each trait which make up this index (Table 3), we could notice that no hybrid stood out considering most traits together (Figure 2 ), and obtained smaller magnitude values compared with the ones of the first season, due to the significant season effect (Reis et al., 2011; Ramalho et al., 2012), showing that the second season was less suitable for the evaluation and selection of the experimental super sweet corn hybrids in Guarapuava.

The hybrid D3-30 x D5-46 showed high standardized means of UEY,
HEY and GY and also showed a good performance in relation to the traits of the ear in both sowing season (Figures 1 and 2), confirming the efficiency of the $I z$ in the selection of this genotype, being an important tool to assist in the selection, according to Reis et al. (2011) and $\mathrm{Wu}$ et al. (2015).

The $Z$ index $\left(I_{Z}\right)$ allowed to discriminate the best hybrids for each sowing season, considering the set of evaluated traits. The traits which most contributed for the $\mathrm{Z}$ index were the ones related to ear yield, grain yield and soluble solids content. The first sowing season was the most favorable to express yield related traits. The experimental hybrid D3-30 x D5-46 showed good performance in both sowing seasons and the hybrid D2-17 x D5-46 stood out in the second sowing season, showing to be promising, being selected due to its high productivity and quality of ear, 
considering the $\mathrm{Z}$ index.

\section{ACKNOWLEDGMENTS}

This study was financed in part by the Coordenação de Aperfeiçoamento de Pessoal de Nível Superior - Brasil (CAPES) - Finance Code 001.

\section{REFERÊNCIAS}

ABE, A; ADELEGAN, CA. 2019. Genetic variability, heritability and genetic advance in shrunken-2 super-sweet corn (Zea mays L. saccharatta) populations. Journal of Plant Breeding and Crop Science 11: 100-105.

AMARAL JÚNIOR, AT; FREITAS JÚNIOR, SP; RANGEL, RM; PENA, GF; RIBEIRO, RM; MORAIS, RC; SCHUELTER, AR. 2010. Improvement of a popcorn population using selection indexes from a fourth cycle of recurrent selection program carried out in two different environments. Genetics and Molecular Research 9: 340-347.

CANDIDO, WS; SILVA, CM; COSTA, ML; SILVA, BEA; MIRANDA, BL; PINTO, JFN; REIS, EF. 2020. Selection indexes in simultaneous increment of yield components in topcross hybrids of green maize. Pesquisa Agropecuária Brasileira 55: e01206.

CRUZ, CD. 2013. GENES - a software package for analysis in experimental statistics and quantitative genetics. Acta Scientiarum Agronomy 35: 271-276.

DOVALE, JC; FRITSCHE-NETO, R; SILVA, PSL. 2011. Índice de seleção para cultivares de milho com dupla aptidão: minimilho e milho verde. Bragantia 70: 781-787.

FAVARATO, LF; SOUZA, JL; GALVÃO, JCC; SOUZA, CM; GUARCONI, RC; BALBINO, JMS. 2016. Crescimento e produtividade do milho-verde sobre diferentes coberturas de solo no sistema plantio direto orgânico. Bragantia 75: 497-506.

FREITAS, ILJ; AMARAL JUNIOR, AT; VIANA, AP; PENA, GF; CABRAL, PS; VITTORAZZI, C; SILVA, TRC. 2013. Ganho genético avaliado com índices de seleção e com REML/BLUP em milho-pipoca. Pesquisa Agropecuária Brasileira 48: 1464-1471.

GURGEL, FL; FERREIRA, DF; SOARES, ACS. 2013. O coeficiente de variação como critério de avaliação em experimentos de milho e feijão. Belém: Embrapa Amazônia
Oriental. 80 p. (Boletim de Pesquisa e Desenvolvimento, 85).

HALLAUER, AR; MIRANDA FILHO, JB. 2010. Quantitative genetics in maize breeding. New York: Springer, 663p.

KUKI, MC; SCAPIM, CA; PINTO, RJB; FIGUEIREDO, AST; CONTRERASSOTO, RI; ERTAGNA, FAB. 2017. Inbreeding depression and average genetic components in green corn genotypes. Ciência Rural 47: e20160024. DOI: https://dx.doi. org/10.1590/0103-8478cr20160024

LIMA, VJ; FREITAS JUNIOR, SP; SOUZA, YP; SILVA, CS; FARIAS, JEC; SOUZA, RF; CHAVES, MM; FEITOSA, JV. 2018. Genetic gain capitalization in the first cycle of recurrent selection in popcorn at Ceará's Cariri. Revista Brasileira de Ciências Agrárias 13: e 5556.

LUZ, JMQ; CAMILO, JS; BARBIERI, VHB; RANGEL, RM; OLIVEIRA, RC. 2014. Produtividade de genótipos de milho doce e milho verde em função de intervalos de colheita. Revista Ceres 32: 163-167.

MACHADO FILHO, GC; NASCIMENTO, IR; SAKAI, TRP; ROCHA, WS; SANTOS, MM. 2018. ${ }^{\circ}$ Brix e produção de espigas de milho verde em função de épocas de adubação nitrogenada. Pesquisa Aplicada e Agrotecnologia 11: 33-41.

MAMEDE, NGMA; CHITARRA, BA; FONSECA, JOM; SOARES, GA; PEREIRA FILHO, AI. 2014. Qualidade do milho doce minimamente processado conservado sob diferentes atmosferas. Engenharia na Agricultura 22: 520-534.

MENDES, FF; RAMALHO; MAP; ABREU, AFB. 2009. Índice de seleção para escolha de populações segregantes. Pesquisa Agropecuária Brasileira 44: 1312-1318.

NITSCHE, PR; CARAMORI, PH; RICCE, WS; PINTO, LFD. 2019. Atlas climático do Estado do Paraná. Londrina: Instituto Agronômico do Paraná.

PEREIRA FILHO, IA; CRUZ, JC; BORGHI, E. 2016. Cultivares de milho-doce. In: PEREIRA FILHO, IA; TEIXEIRA, FF (eds). O cultivo do milho doce. $1^{\mathrm{a}}$ ed. Sete Lagoas: Embrapa Milho e Sorgo, p.55-60.

PERFEITO, DGA; LOPES, MCM; SALOMÃO, LC; SOUZA, MLC; BENETT, CGS; LIMA, BP. 2017. Caracterização pós-colheita de milho doce submetido ao parcelamento de fertirrigação nitrogenada. Brazilian Journal of Food Technology 20: 1-7.

RAMALHO, MAP; ABREU, AFB; SANTOS, JB; NUNES, JAR. 2012. Aplicações da genética quantitativa no melhoramento de plantas autógamas. Lavras: UFLA. 522p.
REIS, CAF; GONÇALVES, FMA; RAMALHO, MAP; ROSADO, AM. 2011. Seleção de progênies de eucalipto pelo índice $Z$ por MQM e Blup. Pesquisa Agropecuária Brasileira 46: 517-523.

SANTOS, PHAD; PEREIRA, MG; TRINDADE, RS; CUNHA, KS; ENTRINGER, GC; VETTORAZZI, JCF. 2014. Agronomic performance of super-sweet corn genotypes in the North of Rio de Janeiro. Crop Breeding and Applied Biotechnology 14: 8-14.

SHIOGA, PS; GERAGE, AC; ARAÚJO, PM; CUSTÓDIO, AAP; BARROS, ASR; GARBUGLIO, DD. 2017. Avaliação estadual de cultivares de milho safra 2016/2017. Instituto Agronômico do Paraná, IAPAR. Boletim Técnico 89. Londrina.

SILVA, IG; CASTOLDI, R; CHARLO HCO; MIRANDA, MS; NUNES, TDC; COSTA, LL; LEMES, EM. 2020a. Prediction of genetic gain in sweet corn using selection indexes. Journal of Crop Science Biotechnology 23: 191-196. DOI: https://doi.org/10.1007/ s12892-019-0334-0

SILVA, MF; MACIEL, GM; FINZI, RR; PEIXOTO, JVM; REZENDE, WS; CASTOLDI, R. 2020b. Selection indexes for agronomic and chemical traits in segregating sweet corn populations. Horticultura Brasileira 38: 71-77. DOI: http://dx.doi. org/10.1590/S0102-053620200111

SOLOMON, KF; MARTIN, I; ZEPPA, A. 2011. Genetic effects and genetic relationships among shrunken (sh2) sweet corn lines and F1 hybrids. Euphytica 185: 385-394.

SOUZA NETO, IL. 2019. Definição de grupos heteróticos em milho superdoce por meio da distância genética e capacidade combinatória. 97p. Botucatu: UNESP. (Ph.D. Thesis).

VENCOVSKY, R. 1978. Herança quantitativa. In: PATERNIANI, E (ed). Melhoramento e produção do milho no Brasil. Campinas: Fundação Cargill, p.122-195.

VIEIRA, RA; ROCHA, R; SCAPIM, CA; AMARAL JUNIOR, AT. 2017. Recurrent selection of popcorn composites UEM-CO1 and UEM-CO2 based on selection indices. Crop Breeding and Applied Biotechnology 17: 266-272.

WU, D; QU, JJ; HAO, X. 2015. Agricultural drought monitoring using MODIS-based drought indices over the USA corn belt. International Journal of Remote Sensing, 36: 5403-5425.

YUWONO, PD; MURTI, RH; BASUNANDA, P. 2017. Heterosis and specific combining ability in sweet corn and its correlation with genetic similarity of inbred lines. Journal of Agricultural Science 9: 245-253. 